\title{
Plant facilitation through mycorrhizal symbiosis is stronger between distantly related plant species
}

\author{
Alicia Montesinos-Navarro ${ }^{1}$, Alfonso Valiente-Banuet ${ }^{2,3}$ and Miguel Verdư ${ }^{1}$ \\ ${ }^{1}$ Centro de Investigaciones sobre Desertificación (CIDE, CSIC-UV-GV), Carretera de Moncada-Naquera Km 4.5, Moncada, Valencia 46113, Spain; ${ }^{2}$ Departamento de Ecología de la \\ Biodiversidad, Instituto de Ecologia, Universidad Nacional Autonoma de Mexico, A.P. 70-275, Mexico, D. FC. P. 04510, Mexico; ${ }^{3}$ Centro de Ciencias de la Complejidad, Ciudad Universitaria, \\ Universidad Nacional Autonoma de México, Mexico, D. F04510, México
}

Author for correspondence: Alicia Montesinos-Navarro Tel: +34963424126

Email: ali.montesinos@gmail.com

Received: 17 May 2019

Accepted: 29 June 2019

New Phytologist (2019)

doi: $10.1111 / \mathrm{nph} .16051$

Key words: biotic interactions, mycorrhizae, phylogenetic distance, plant facilitation, symbiosis.

\section{Summary}

- The tendency of closely related plant species to share natural enemies has been suggested to limit their co-occurrence and performance, but we lack a deep understanding on how mutualistic interactions such as the mycorrhizal symbiosis affect plant-plant interactions depending on the phylogenetic relatedness of the interacting plants. We hypothesise that the effect of the mycorrhizal symbiosis on plant-plant facilitative interactions depends on the phylogenetic distance between the nurse and facilitated plants.

- A recently published meta-analysis compiled the strength of plant facilitative interactions in the presence or absence (or reduced abundance) of mycorrhizal fungi. We use phylogenetically informed Bayesian linear models to test whether the effect size is influenced by the phylogenetic distance between the plant species involved in each plant facilitative interaction.

- Conspecific facilitative interactions are more strongly enhanced by mycorrhizal fungi than interactions between closely related species. In heterospecific interactions, the effect of the mycorrhizal symbiosis on plant facilitation increases with the phylogenetic distance between thenurseandfacilitated plantspecies.

- Our result showing that the effect of mycorrhizal symbiosis on the facilitation interactions between plants depends on their phylogenetic relatedness provides new mechanisms to understand how facilitation is assembling ecological communities.

\section{Introduction}

The mechanisms shaping plant communities have intrigued ecologists since the origins of the discipline (Watt, 1947; Rolhauser \& Pucheta, 2017). A greater intensity of negative interactions between close relatives has been traditionally invoked to explain plant community structure (Valiente-Banuet \& Verdú, 2013). Janzen (Janzen, 1970) and Connell (Connell, 1971) formally hypothesised that host-specific natural enemies can maintain tree species diversity in tropical forests by reducing the seedling performance near the parent tree (Bagchi et al., 2014). This idea was expanded by Webb et al. (2006) shifting from a single conspecific/heterospecific dichotomy to a continuous gradient of phylogenetic relatedness. Since then, there has been an increasing interest on using phylogenetic relatedness to predict the outcome of plant interactions (Verdu et al., 2012). Several authors have tested whether seedling performance is negatively correlated with the phylogenetic relatedness between neighbours (Castillo et al., 2010; Metz et al., 2010; Paine et al., 2012; Lebrija-Trejos et al., 2014), usually appealing to enemy-mediated mechanisms. The probability of sharing an enemy will decrease with the phylogenetic distance between hosts (Parker \& Gilbert, 2004; Novotny et al., 2006; Gilbert \& Webb, 2007), as closely related species may share traits that enhance their vulnerability to the same enemies (Mitter et al., 1991; Gomez et al., 2010). Although more rarely considered, positive interactions like plant facilitation and the mycorrhizal symbiosis, have also shown to follow a phylogenetic pattern that can be used to explain plant community structure (Montesinos-Navarro etal., 2012a, 2016a, 2018a).

Mycorrhizal symbiosis can influence facilitative interactions among plants (Callaway et al., 2001; Callaway, 2007; Montesinos-Navarro et al., 2012a), defined as plant-plant interactions in which one plant (facilitated) gets a benefit from growing associated with another, without resulting in any disadvantage for the latter (nurse) (Callaway, 2007). Contrary to enemies-mediated mechanisms shaping plant co-occurrence, the presence of conspecifics or closely related species may counterbalance competition between close relatives by increasing the abundance of shared symbionts in the rhizosphere. However, the expected effect of the mycorrhizal symbiosis on facilitative plant interactions might differ between conspecific and heterospecific plant interactions if species specificity in plant-fungi interactions exists. Although there is no consensus on whether closely related plant species tend to interact with similar mycorrhizal symbionts (Montesinos-Navarro et al., 2012a; Reinhart \& Anacker, 2014; Veresoglou \& Rillig, 2014; Chen et al., 2017), it is increasingly 
recognised that these interactions are more species specific than previously considered (Gollotte et al., 2004; Tedersoo et al., 2008; Montesinos-Navarro et al., 2012b, 2018a). Therefore, facilitative interactions between conspecific plants, unlike those between heterospecific plants, might provide a higher abundance of shared mycorrhizal fungi potentially increasing seedling survival (Dickie et al., 2002; Teste et al., 2009; Booth \& Hoeksema, 2010). A particular case of conspecific interaction is facilitation between individuals with different degrees of kinship. Seedlings can preferentially transfer carbon (C) to their full-siblings through shared common mycorrhizal fungi (Pickles etal., 2017), potentially mediated by the recognition of specific root exudates that can allow the detection of offspring's roots (Biedrzycki etal., 2010). At present it is unknown whether kin recognition mechanisms can be also at work in plant-plant interactions.

The effect of the mycorrhizal symbiosis on the outcome of heterospecific facilitation can be influenced by the phylogenetic distance between the nurse and facilitated species through two main factors: (1) mycorrhizal fungi can provide dissimilar resources to distantly related host plants, and (2) mycorrhizal fungi can enhance interplant resource transfer between distant relatives (Montesinos-Navarro et al., 2018a). Major plant lineages differ in their ability for resource acquisition (Cornwell et al., 2014), potentially demanding different resources to their associated fungal community. In addition, distantly related species can create steeper resource gradients that promote nutrient exchange between them (Montesinos-Navarro et al., 2017), in part mediated by shared mycorrhizal fungi that allow nutrient movement from nutrient-rich to nutrient-poor environments (Bethlenfalvay et al., 1991; Frey \& Schfiepp, 1993). However, a myriad of different mechanisms mediated by mycorrhizae may also affect plant interactions (Montesinos-Navarro et al., 2018a), blurring the expected pattern of enhanced facilitation with phylogenetic distance between plant species. Nevertheless, in nature, there is a pervasive pattern of successful facilitation between distantly related plants (Valiente-Banuet \& Verdú, 2007, 2008).

In this study, we used data compiled in a recently published meta-analysis to hypothesise that the effect of the mycorrhizal symbiosis on plant-plant facilitative interactions depends on the phylogenetic distance between the nurse and facilitated plants. We specifically tested whether the effect of mycorrhizal symbiosis on plant facilitation: (1) differs between conspecific and heterospecific interactions; and (2) depends on the phylogenetic distance between the nurse and facilitated species.

\section{Materials and Methods}

\section{Database compilation}

We used the data from a meta-analysis focused on the effect of the mycorrhizal symbiosis on plant biomass and nutrient content of facilitated plants (Montesinos-Navarro et al., 2018b). This metaanalysis provides a thorough description of the data in terms of distribution of data across ecosystems, mycorrhizal type (arbuscular or ectomycorrhiza), performance variables measured and the full reference of the papers used. The response variable compiled was the performance of the facilitated plant measured in 'control' and 'treated' environments, the 'control' environment being places where mycorrhizal abundance was higher than in its 'treated' pair. Two more papers with relevant data were found and added to the database (Borchers \& Perry, 1990; Onguene \& Kuyper, 2002). Finally, we discarded cases in which the identity of the nurse species was unknown. The database resulted in 215 cases from 19 studies (Supporting Information Table S1). We also assessed whether the database used can be considered a representative sample of the phylogenetic distances between species in which facilitative interactions have been reported. To do so, we compiled the pairwise interactions reported in two revisions of plant facilitative interactions (Bonanomi et al., 2011; de Toledo Castanho et al., 2015) that included 2127 facilitative interactions between 1650 plant species, and estimated the phylogenetic distances between each pair using the methodology described in the following section. As shown in Fig. S1, the distribution of the phylogenetic distances in our database matched very well that in the overall database of facilitative interactions, indicating that our database is a representative sample of the phylogenetic distances between plants involved in facilitative interactions published in the literature. Future research on facilitation covering under-represented phylogenetic distances will help to support or refute the patterns found here.

\section{Phylogenetic distances between plant species}

The phylogenetic relationships among the plant species were generated with the R function S.PhyloMAKer (Qian \& Jin, 2016). It uses the PhytoPhylo backbone megaphylogeny, which is an updated and expanded version of the time-calibrated angiosperm species-level phylogeny (Zanne et al., 2014). The community phylogeny was produced by matching the family names of the plant species in our database with those in the backbone phylogeny, using the R package APE (Paradis et al., 2004). Then, we used a database compiling expert-knowledge in molecular time estimates of divergence among taxa (TimeTree: http:// www.timetree.org/) to extract the confidence interval of the age estimate for each node, and used it to calibrate the tree by performing a branch smoothing using the 'chronos' function in the APE package implemented in the software R v.3.2.2 (Paradis etal., 2004; R Core Team, 2015). To account for the uncertainty of age estimates, we iterated the branch smoothing 100 times to generate 100 phylogenies. The branch lengths of those phylogenies were constrained by the confidence interval of each node's age. For each of the 100 phylogenies, the phylogenetic distance between the nurse and facilitated species was obtained using the 'cophenetic' function in the APE package (Paradis etal., 2004).

\section{Effect size}

We calculated the facilitated species' performance under controlled or treated conditions to assess the effect size using Hedges' $\mathrm{g}$ standardised mean difference (Hedges, 1981), and used largesample approximation to compute the sampling variances (Viechtbauer, 2015). Hedges' g can be defined as the difference between the mean of the control and treated individuals, divided 
by the pooled and weighted standard deviation. This metric quantifies the increment (if positive) or decrement (if negative) in the benefits that the facilitated plant obtained from its nurse in an environment with a higher abundance of mycorrhizal fungi. Hedges' $g$ metric and the sampling variance for each case were calculated using the function ESCAL in the package METAFOR (Viechtbauer, 2015) in R v.3.5.2 (R Core Team, 2015).

\section{Statistical analyses}

The estimated $I^{2}$ index of heterogeneity (Higgins \& Thompson, 2002) reported in Montesinos-Navarro et al. (2018b) showed that the effect size's variation cannot be fully explained by measurement error. Therefore, other sources of variation can be explored, and we tested whether the effect size of mycorrhizae on plant facilitative interactions can be explained by the phylogenetic distance between the nurse and the facilitated species. First, we assessed whether the effect of mycorrhizal fungi on plant facilitative interactions was similar in conspecific and heterospecific interactions at different phylogenetic distances. We classified the phylogenetic distances between interacting plant species into: conspecifics (i.e.phylogenetic distance 0Myr), species within the same clade (monocots, dicots or gymnosperms) (<300 Myr), species from close clades (one monocot and one dicot) (300$500 \mathrm{Myr}$ ), and species from distant clades (one gymnosperm and one angiosperm) (> 500 Myr). Then we fitted a Bayesian linear model with the effect size as the response variable and the categorised phylogenetic distances as the explanatory variable, and post-hoc tests between each pair of relatedness categories to assess differences between them. Second, we tested whether the effect size of mycorrhizae on plant facilitative interactions can be explained by the phylogenetic distance between the nurse and the facilitated species. We considered only heterospecific interactions if conspecific and heterospecific interactions showed different patterns in the previous test. For this, we fitted a phylogenetically informed Bayesian linear model with the effect size as the response variable and the phylogenetic distance between the nurse and the facilitated species as explanatory variable. Previous studies suggested that the phylogenetic patterns observed in plant facilitative interactions were more usually driven by the facilitated than by the nurse species (Verdu et al., 2010, 2012). Therefore, we informed the model with the phylogenetic relationships among facilitated species, and included the original paper and the identity of the facilitated species as random factors. We also tested for the significance of considering the phylogeny of the facilitated plants by repeating the analyses without considering it, and compared the deviance information criterion (DIC) of both models. For each model, we reported the probability that the estimate was higher than 0 , expressed in $\%$, and its credibility interval (CI). In each Bayesian model we used a prior with an expected mean value of $\mathrm{mu}=0$ and a (co)variance matrix of $V=I 91 \mathrm{e}+10$, where $\mathrm{I}$ is an identity matrix of appropriate dimension. Models were weighted by the inverse of the sampling variance of each case. The settings were chosen to store at least 1000 MCMC (Markov chain Monte Carlo) iterations with an autocorrelation between successive stored iteration $<0.1$. This was usually reached using 13000 iterations, thinned every 10 and discarding a burn-in period of $25 \%$ of the iterations. To account for the uncertainty in node age estimates, each model was repeated 100 times using, in turn, each of the 100 phylogenies. Then, the $100 \mathrm{MCMC}$ were integrated by concatenating a random sample of estimates across all of them. Bayesian models were performed using the package MCMCGLMm (Hadfield, 2010). Only two records in our database involve a plant-plant interaction with a phylogenetic distance above 500 Myr mediated by arbuscular mycorrhizal fungi. To discard that the phylogenetic patterns could be confounded with the type of mycorrhizal fungi, we also tested for the relationship considering only plant-plant interactions mediated by ectomycorrhizal fungi.

\section{Data availability}

The data used in this study is provided as Table S1.

\section{Results}

Our database compiled 215 cases published in 19 studies, in which the pairwise interactions of 26 nurse species and 21 facilitated species were reported (Table $\mathrm{S} 1$ ). The phylogenetic distance between the nurse and the facilitated species (i.e. twice the time since the two species diverge from their common ancestor) in the cases of heterospecific facilitation ranged from 29.1 Myr to 669 Myr.

The effect of the mycorrhizal symbiosis on plant facilitation depended on whether the interaction occurred between conspecifics or heterospecifics across different phylogenetic distances between them. The mycorrhizal symbiosis enhanced facilitation between conspecifics (mean $=1.25 ; 99 \% \mathrm{CI}=0.85,1.67)$, while the effect size between heterospecifics belonging to the same clade (either within monocots, dicots or gymnosperms) had a mean of -0.29 , with a low probability of being different from zero $61 \%$ $\mathrm{CI}=-0.60,-0.06)$. Between heterospecifics from distant clades (a gymnosperm with an angiosperm), the mycorrhizal symbiosis enhanced facilitation even more than between conspecifics ( mean $=2.38 ; 99 \% \mathrm{CI}=1.55,3.35$ ). There was not a significant difference between conspecific interactions and those between species from close clades $($ mean $=0.96 ; 99 \% \mathrm{CI}=0.23,1.60)$ (Fig. 1). When we considered only interactions mediated by ectomycorrhizal fungi, the same pattern emerged (conspecifics: mean $=1.31 ; 99 \% \mathrm{CI}=0.97,1.61$; heterospecifics in the same clade: mean $=-0.41 ; 99 \% \mathrm{CI}=-0.89,-0.02$; heterospecifics in distant clades: mean $2.35 ; 99 \% \mathrm{CI}=1.76,3.01)$. None of the interactions between plant species from close clades was mediated by ectomycorrhizal fungi.

The effect size tended to be stronger in cases in which heterospecific interacting species were distantly related (Fig. 2). The Bayesian models showed that the effect size of mycorrhizae on plant facilitation significantly increased with the phylogenetic distance between the nurse and the facilitated species (mean effects size $=0.009 ; 99 \% \mathrm{CI}=0.005,0.01)$. The phylogenetic relationship between the facilitated species did not contribute significantly to improve the model accuracy, shown by the similar 


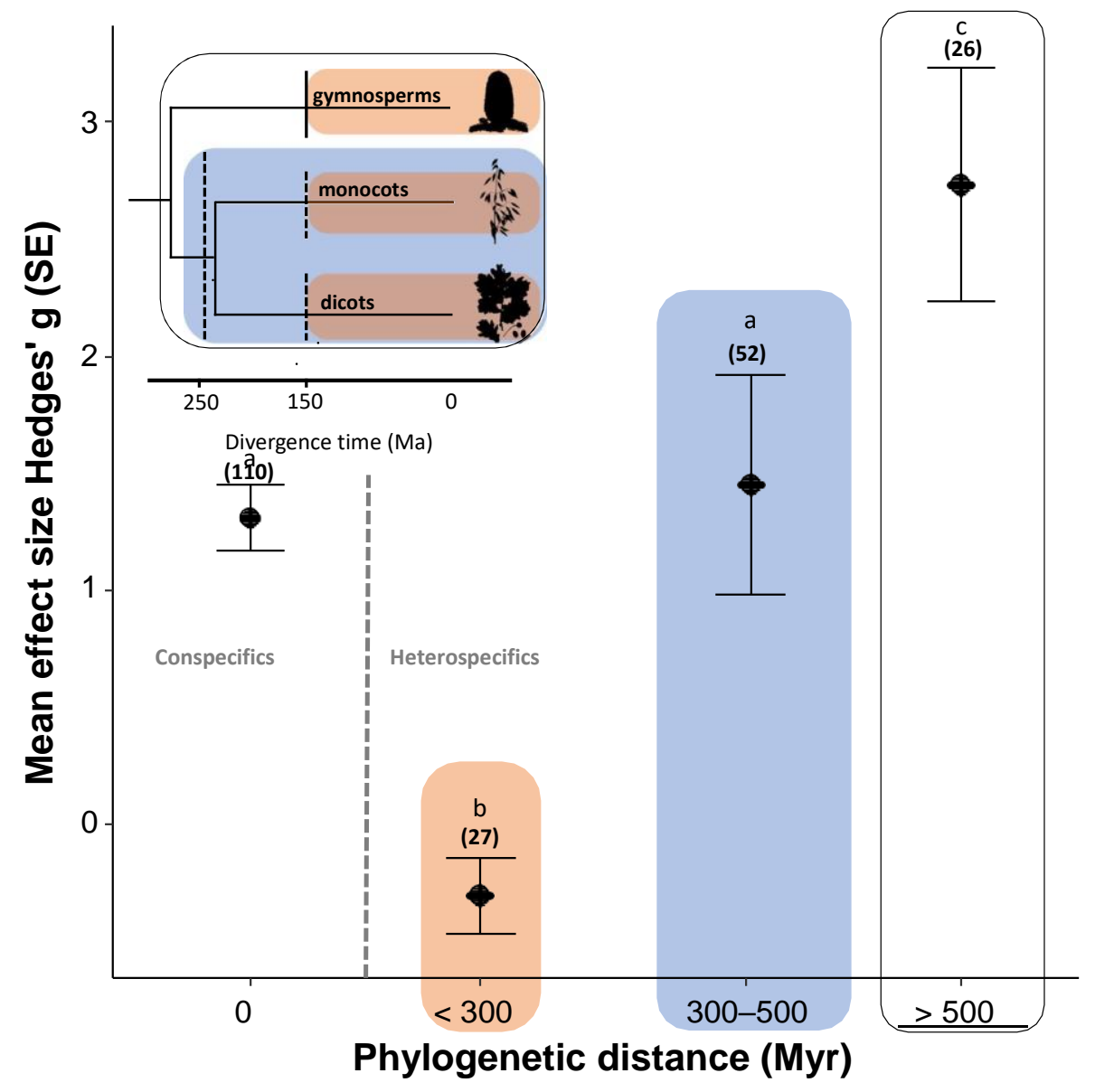

Fig. 1 The effect of mycorrhizal symbiosis on conspecific and heterospecific plant facilitation with different phylogenetic distances between the two plant species involved. Conspecific relationships are presented on the left and heterospecific interactions on the right of the dashed line. Heterospecific interactions are classified as: species within the same clade (either within monocots, dicots or gymnosperms) (<300 Myr), species from close clades (one monocot and one dicot) (300-500 Myr), and species from distant clades (one gymnosperm and one angiosperm) (> $500 \mathrm{Myr}$ ). In the inset, divergence time between species is expressed as millions of years ago (Ma) and orange, blue and white rectangles group pairs of species within clade, from close and distant clades, respectively. The icons represent from top to bottom: gymnosperms, monocots (e.g. Poaceae), and dicots (e.g. Fagaceae). Significant differences in the mean effect size (mean \pm SE) between interactions across phylogenetic distances, quantified as Hedges' g standardised mean difference, are represented by different letters. Sample size in brackets. Phylogenetic distance between two species in millions of years (Myr) is twice the time since they diverged from their mostcommon ancestor.
DIC found in the model considering (DIC = 352) and not considering $(\mathrm{DIC}=354)$ the phylogeny. Therefore, there are not specific clades driving the pattern observed. The positive relationship between the phylogenetic distance between the nurse and facilitated species and effect size was also maintained with $86 \%$ of probability when only the interactions mediated by ectomycorrhizal fungi were taken into account ( mean effects size $=0.004$; $86 \% \mathrm{CI}=0.001,0.007)$.

\section{Discussion}

Our meta-analysis revealed that the enhancement of plant facilitation by the mycorrhizal symbiosis increased with the phylogenetic distance between the heterospecific interacting plants. Plants facilitated by conspecifics benefit more from a higher abundance of mycorrhizal fungi than plants facilitated by closely related heterospecifics (i.e. both species belonging to the same monocot, dicot or gymnosperm clades). In heterospecific interactions, the benefits gained by the facilitated plant increases when the plant is growing with a distant relative, and this pattern is independent of specific families or clades.

In our database and in plant facilitative interactions in general, the most common gymnosperms belonged to the Pinaceae family (57\% of the interactions involving gymnosperms reported in Bonanomi et al., 2011; de Toledo Castanho et al., 2015). This results in a predominance of Pinaceae, when one of the interacting plants is a gymnosperm (i.e. interactions between distant relatives in our database), which are usually associated with ectomycorrhizal fungi. The enhancement of plant facilitation by the mycorrhizal symbiosis between distant relatives might be influenced by the predominance of ectomycorrhizae in these particular interactions. However, this seems unlikely considering that this relationship is maintained when only interactions mediated by ectomycorrhizal fungi are considered across all phylogenetic distances. Unfortunately, facilitative interactions mediated by arbuscular mycorrhizal fungi between distant relatives are less frequent in the literature, this situation prevents testing for the correlation that considers only arbuscular mycorrhizal fungi-mediated interactions. Further research on arbuscular mycorrhizal gymnosperms will allow the comparison of its effect on both close and distant relatives within this clade, due to a wider range of divergence time between species in thisclade.

The contrasting pattern between interactions involving conspecifics and closely related plant species suggested that different mechanisms through which the mycorrhizal symbiosis enhances plant facilitation are acting. Several mechanisms that might specifically enhance facilitation between conspecifics but not closely related species are: (1) shared mutualists, (2) shared defenses, and (3) showed kinship selection. Considering that certain specificity in plant-mycorrhizal fungi interactions is 


\section{Facilitated species}

\section{Nurse species}

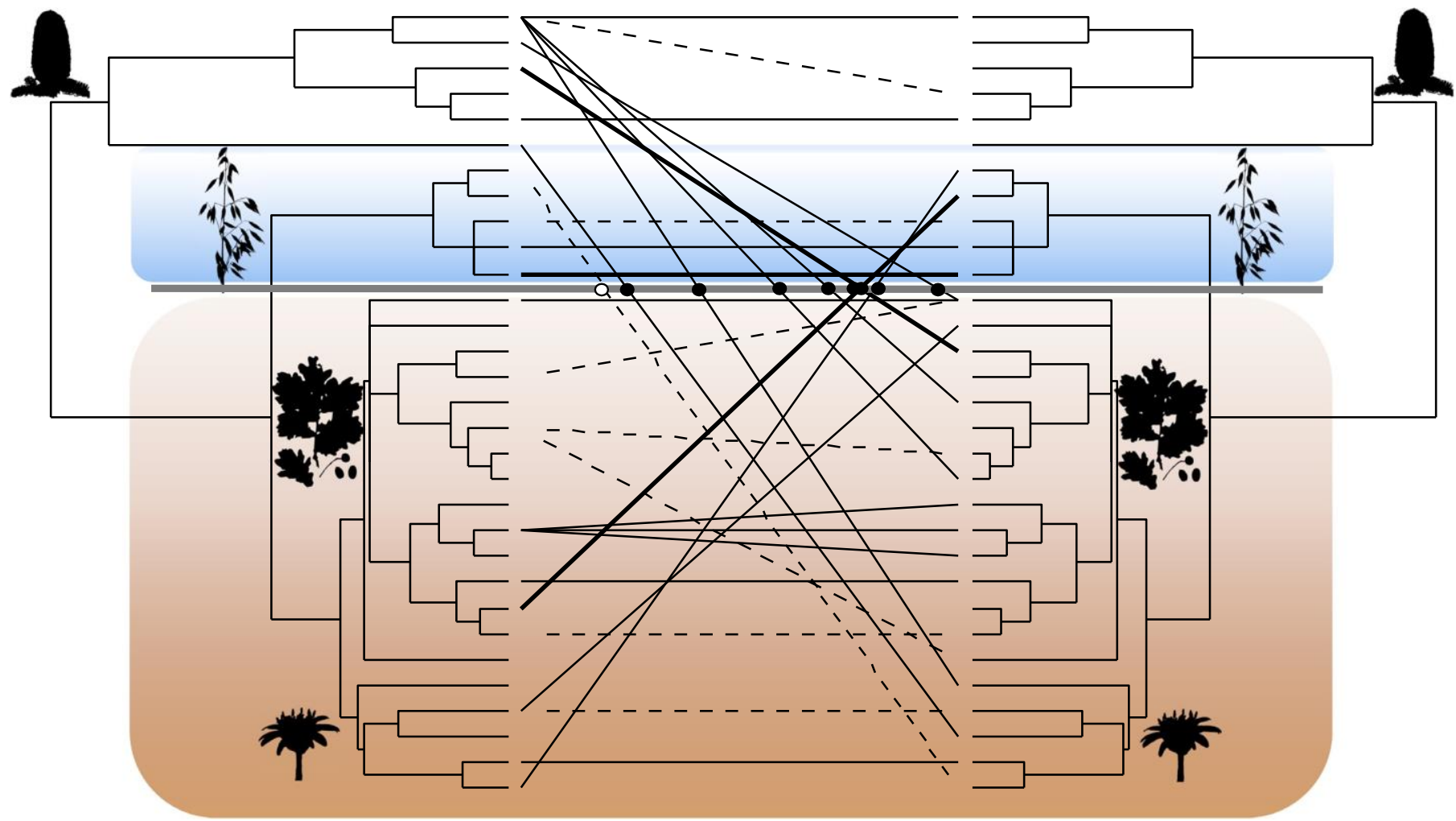

Fig. 2 The effect size of mycorrhizal symbiosis on plant facilitation interactions between plant species with different phylogenetic distance. The phylogenetic trees represent the relationship between all the plant species included in the meta-analysis (full species names are presented in Supporting Information TableS1). Facilitated and nurse plants are presented on the left and right trees, respectively. The icons identify from top to bottom: gymnosperms, monocots (i.e. Poaceae), and two families of dicots (i.e. Fagaceae and Asteraceae). Links between the two phylogenies represent the presence of a given pairwise plant-plant interaction in our data set, and the thickness of the links represent the logarithm of the arithmetic weighted mean of the effect size across all the cases where the specific plant-plant interaction was measured. Solid and dashed lines represent positive (facilitation is enhanced by mycorrhizae) and negative (facilitation is reduced by mycorrhizae) values, respectively. Lines crossing the grey horizontal bar correspond to interactions between distant relatives, as one of the two species involved is a dicot (highlighted in orange), and the other is outside that clade. Black points highlight facilitation between distant relatives enhanced by mycorrhizae (i.e. solid links crossing the grey bar) and white points represent facilitation between distant relatives reduced by mycorrhizae (i.e. dashed links crossing the grey bar). The predominance of black vs white points indicates that mycorrhizae promote facilitation between distant relatives (see the Results section for statistical tests).

increasingly recognised, both in arbuscular and ectomycorrhizal fungi (Gollotte et al., 2004; Tedersoo et al., 2008; MontesinosNavarro etal., 2012b, 2018a), conspecific plants will provide the same symbionts with which the facilitated plant interacted, therefore increasing the abundance of symbionts in the shared rhizosphere. Another potential mechanism can be influenced by mycorrhizal protection against pathogens: ectomycorrhiza may protect the external layers of the roots with their structures while arbuscular mycorrhizae can trigger systemic defense mechanisms (Pozo \& Azcon-Aguilar, 2007; Bennett et al., 2017). Mycorrhizal symbiosis, especially ectomycorrhiza, has been suggested to reduce conspecific competition due to pathogen protection, with closely related plant species having a similar sensitivity to conspecific/heterospecific competition (Bennett et al., 2017). Finally, other mechanisms affecting only conspecific interactions are those related to the facilitation associated with kinship. Seedlings connected through ectomycorrhizal fungi can transfer a threefold amount of ${ }^{13} \mathrm{C}$ to full-siblings than to nonsibling pairs, potentially mediated by root exudates (Pickles et al., 2017).
Plants can also recognise kin neighbours and horizontally reoriented leaf growth to avoid competition mediated by the perception of the vertical red/far-red light and blue light profiles (Crepy $\&$ Casal, 2015). Moreover, volatiles have been suggested to allow self-recognition, inducing a higher resistance to herbivory when volatile cues are emitted from genetically identical cuttings; this could be a first step towards kin recognition (Karban \& Shiojiri, 2009; Karban et al., 2013). Further research is needed to unravel the relative contribution of each mechanism to explain the positive effects of mycorrhizae on conspecific facilitation.

Regarding heterospecific interactions, our model estimated that the effect size of the mycorrhizal symbiosis on plant facilitation will increase by 0.9 if the phylogenetic distance between the nurse and the facilitated species was $100 \mathrm{Myr}$ greater (estimate of the slope $=0.009$ effect size increase per year). To show the biological meaning of this increase in effect size, we will exemplify it using total plant biomass. The mean and averaged standard deviations of the control plants' biomass were $3.2 \mathrm{~g}$ and $2.8 \mathrm{~g}$, respectively. An effect size of 0 implied that both control and treated 
plants had a similar biomass, therefore $3.2 \mathrm{~g}$. Then, based on a simplified definition of the Hedges' $g$ (effect size $=$ mean control - mean treated/standard deviation) we can calculate the estimated plant biomass of the treated plants if the effect size was 0.9 (i.e. $3.2-(0.992 .8)=0.68 \mathrm{~g})$. Therefore, we can state that when mycorrhizal symbiosis is present, the facilitated plant biomass can increase by $78 \%$ when growing with a nurse that is $100 \mathrm{Myr}$ more phylogenetically distant to it than the other $((3.2-0.68) /$ $3.29100)=78 \%$ ).

Some mechanisms could result in a mycorrhizae-mediated enhancement of facilitative interactions between distant relatives: (1) nutrient transfer through common mycorrhizal networks; and (2) reduced competition. Common mycorrhizal networks can connect plant roots of different plant species and families (Selosse et al., 2006). These connections can allow the transfer of a wide diversity of compounds between plants, including nutrients such as C, N and P (He et al., 2009; Ren et al., 2013; Song et al., 2015). Nutrient transfers from rich-nutrient to poor-nutrient plants (Bethlenfalvay et al., 1991; Frey \& Schfiepp, 1993). The foliar content of nutrients such as $\mathrm{N}$ and $\mathrm{P}$ are likely to be phylogenetically conserved, as several traits involved in nutrient acquisition are strongly phylogenetically conserved (i.e. $\mathrm{N}_{2}$ fixation through rhizobium symbiosis or clustered roots that improve P uptake) (Stock \& Verboom, 2012; Cornwell et al., 2014; Yang et al., 2015). This can result in steep nutrient gradients between distantly related plant species that, in the case for $\mathrm{N}$, has been shown to result in an increase in $\mathrm{N}$ transfer among distant relatives (Montesinos-Navarro etal., 2017). Although this transfer can occur through different pathways besides mycorrhizal connections, a high abundance of mycorrhizal fungi in the soil has been shown to promote nutrient transfer between plants involved in facilitative interactions (Montesinos-Navarro et al., 2016b).

Reduced competition between distant relatives is thought to be the most common mechanism that allows plants to coexist (Valiente-Banuet \& Verdu, 2007; Castillo et al., 2010; Pausas \& Verdu, 2010). A balance between the benefits of reduced competition between distant relatives and a higher abundance of shared mycorrhizal fungi between conspecific can result in the observed pattern of similar benefits for the facilitated plant when they grow with conspecific and with intermediate distant relatives. Interactions with species from close clades might lack the benefits of conspecific interactions, and it is currently under debate whether plant-mycorrhizal fungi interactions are evolutionarily conserved (Montesinos-Navarro etal., 2012a; Reinhart \& Anacker, 2014; Veresoglou \& Rillig, 2014; Chen et al., 2017). In addition, a contrasting effect of plant-plant interactions along gradients can be due to shifts in the net effects of each component, and partitioning these net effects into each component can contribute to understanding the underlying mechanisms (Michalet et al., 2014). The presence of the mycorrhizal symbiosis (i.e. control cases) could increase the benefits between distant relatives due to poorer performance of the plants without mycorrhizae (i.e. treated) or due to increased performance of the plants with mycorrhizae (i.e. control). Further experiments exposing the same target species across a wide phylogenetic context (i.e. phylogenetic distances between the nurse and facilitated between 300 and $669 \mathrm{Myr}$ ) would be necessary to disentangle the underlying mechanisms.

Other mechanisms linking mycorrhizal symbiosis and plant facilitation might now be revisited under the plant phylogenetic perspective. For example, the well known mechanism in which mycorrhizal fungi promote heterospecific facilitation is the provision of inoculum, including reduced costs of establishing an extensive fungal mycelium (Newman, 1988). Through this mechanism, pioneer plants facilitate later successional plants (Dickie etal., 2004; Nara \& Hogetsu, 2004; Nara, 2006), a pattern that usually occur between distantly related species (Verdu et al., 2009).

In short, this meta-analysis reveals that mycorrhizal symbiosis enhances facilitative interactions between conspecifics and also between distant relatives, and proposes some mechanisms consistent with this observation. Our results can contribute to a better understanding of the phylogenetic patterns imprinted by facilitation, an interaction, increasingly recognised as a relevant mechanism structuring plant communities.

\section{Acknowledgements}

AMN was supported by a postdoctoral contract from the Spanish Ministry of Economy and Competitiveness (FPDI-2013-16266; IJCI-2015-23498). Financial support was provided by Early Career Project Grant from the BES (3975-4849), the Spanish Ministry of Economy and Competitiveness (RTI2018-099672-JI00), PAPIIT-DGAPA-UNAM (IN-213414-3; IN-210117), the regional government Generalitat Valenciana (PROMETEO/ 2016/021, GV/2016/187), Red CYTED (P417RT0228) and Ecometas excellence network (CGL2016-81706-REDT).

\section{Author contributions}

AM-N, AV-B and MV planned and designed the research. AM$\mathrm{N}$ compiled and analysed the data, and wrote the first draft of the manuscript. All authors contributed to the last version of the manuscript.

\section{References}

Bagchi R, Gallery RE, Gripenberg S, Gurr SJ, Narayan L, Addis CE, Freckleton RP, Lewis OT. 2014. Pathogens and insect herbivores drive rainforest plant diversity and composition. Nature 506: 85-88.

Bennett JA, Maherali H, Reinhart KO, Lekberg Y, Hart MM, Klironomos J. 2017. Plant-soil feedbacks and mycorrhizal type influence temperate forest populationdynamics. Science 355:181-184. 
Bethlenfalvay GJ, Reyes-Solis MG, Camel SB, Ferrera-Cerrato R. 1991. Nutrient transfer between the root zones of soybean and maize plants connected by a common mycorrhizal mycelium. Physiologia Plantarum 82: 423-432.

Biedrzycki ML, Jilany TA, Dudley SA, Bais HP. 2010. Root exudates mediate kin recognition in plants. Communicative \& Integrative Biology 3: 28-35.

Bonanomi G, Incerti G, Mazzoleni S. 2011. Assessing occurrence, specificity, and mechanisms of plant facilitation in terrestrial ecosystems. Plant Ecology 212: $1777-1790$

Booth MG, Hoeksema JD. 2010. Mycorrhizal networks counteract competitive effects of canopy trees on seedling survival. Ecology 91: 2294-2302.

Borchers SL, Perry DA. 1990. Growth and ectomycorrhiza formation of Douglas-fir seedlings grown in soils collected at different distances from pioneering hardwoods in southwest Oregon clear-cuts. Canadian Journal of Forest Research 20: 712-721.

Callaway RM. 2007. Positive interactions and interdependence in plant communities. Dordrecht, the Netherlands: Springer.

Callaway R, Newingham B, Zabinski CA, Mahall BE. 2001. Compensatory growth and competitive ability of an invasive weed are enhanced by soil fungi and native neighbours. Ecology Letters 4: 429-433.

Castillo JP, Verdu M, Valiente-Banuet A. 2010. Neighborhood phylodiversity affects plant performance. Ecology 91: 3656-3663.

Chen L, Zheng Y, Gao C, Mi X-C, Ma K-P, Wubet T, Guo L-D. 2017. Phylogenetic relatedness explains highly interconnected and nested symbiotic networks of woody plants and arbuscular mycorrhizal fungi in a Chinese subtropical forest. Molecular Ecology 26: 2563-2575.

Connell JS. 1971. On the role of natural enemies in preventing competitive exclusion in some marine animals and in rain forest trees. In: Boer PJD, Gradwell GR, eds. Dynamics of populations. Wageningen, the Netherlands: Pudoc, $298-312$.

Cornwell WK, Westoby M, Falster DS, FitzJohn RG, O’Meara BC, Pennell MW, McGlinn DJ, Eastman JM, Moles AT, Reich PB et al. 2014. Functional distinctiveness of major plant lineages. Journal of Ecology 102: 345-356.

Crepy MA, Casal JJ. 2015. Photoreceptor-mediated kin recognition in plants. New Phytologist 205: 329-338.

Dickie IA, Guza RC, Krazewski SE, Reich PB. 2004. Shared ectomycorrhizal fungi between a herbaceous perennial (Helianthemum bicknellii) and oak (Quercus) seedlings. New Phytologist 164: 375-382.

Dickie IA, Koide RT, Steiner KC. 2002. Influences of established trees on mycorrhizas, nutrition, and growth of Quercus rubra seedlings. Ecological Monographs 72: 505-521.

Frey B, Schfiepp H. 1993. A role of vesicular-arbuscular (VA) mycorrhizal fungi in facilitating interplant nitrogen transfer. Soil Biology and Biochemistry 25: 651-658.

Gilbert GS, Webb CO. 2007. Phylogenetic signal in plant pathogen-host range. Proceedings of the National Academy of Sciences, USA 104: 4979-4983.

Gollotte A, van Tuinen D, Atkinson D. 2004. Diversity of arbuscular mycorrhizal fungi colonising roots of the grass species Agrostis capillaris and Lolium perenne in a field experiment. Mycorrhiza 14: 111-117.

Gomez JM, Verd'u M, Perfectti F. 2010. Ecological interactions are evolutionarily conserved across the entire tree of life. Nature 465: 918-921.

Hadfield JD. 2010. MCMC methods for multi-response generalized linear mixed models: the MCMCglmm R package. Journal of Statistical Software 33:1-22.

He X, Xu M, Qiu GY, Zhou J. 2009. Use of ${ }^{15} \mathrm{~N}$ stable isotope to quantify nitrogen transfer between mycorrhizal plants. Journal of Plant Ecology 2: 107118.

Hedges LV. 1981. Distribution theory for glass's estimator of effect size and related estimators. Journal of Educational Statistics 107-128.

Higgins J, Thompson SG. 2002. Quantifying heterogeneity in a meta-analysis. Statistics in Medicine 21: 1539-1558.

Janzen DH. 1970. Herbivores and the number of tree species in tropical forests. American Naturalist 104: 501-528.

Karban R, Shiojiri K. 2009. Self-recognition affects plant communication and defense. Ecology Letters 12: 502-506.

Karban R, Shiojiri K, Ishizaki S, Wetzel WC, Evans RY. 2013. Kin recognition affects plant communication and defence. Proceedings of the Royal Society of London. Series B, Biological Sciences 280: 20123062.
Lebrija-Trejos E, Wright SJ, Hernandez A, Reich PB. 2014. Does relatedness matter? Phylogenetic density-dependent survival of seedlings in a tropical forest. Ecology 95: 940-951.

Metz MR, Sousa WP, Valencia R. 2010. Widespread density-dependent seedling mortality promotes species coexistence in a highly diverse Amazonian rain forest. Ecology 91: 3675-3685.

Michalet R, Sch6b C, Lortie CJ, Brooker RW, Callaway RM. 2014. Partitioning net interactions among plants along altitudinal gradients to study community responses to climate change. Functional Ecology 28: 75-86.

Mitter C, Farrell B, Futuyma DJ. 1991. Phylogenetic studies of insect-plant interactions: insights into the genesis of diversity. Trends in Ecology \& Evolution 6: 290-293.

Montesinos-Navarro A, Segarra-Moragues JG, Valiente-Banuet A, Verdu M. 2012a. Plant facilitation occurs between species differing in their associated arbuscular mycorrhizal fungi. New Phytologist 196: 835-844.

Montesinos-Navarro A, Segarra-Moragues JG, Valiente-Banuet A, Verd’u M. 2012b. The network structure of plant-arbuscular mycorrhizal fungi. New Phytologist 194:536-547.

Montesinos-Navarro A, Segarra-Moragues JG, Valiente-Banuet A, Verdu M. 2016a. Fungal phylogenetic diversity drives plant facilitation. Oecologia 181: 533-541.

Montesinos-Navarro A, Valiente-Banuet A, Verdu M. 2018a. Processes underlying theeffect of mycorrhizal symbiosis on plant-plantinteractions. Fungal Ecology 40: 98-106.

Montesinos-Navarro A, Valiente-Banuet A, Verdu M. 2018b. Mycorrhizal symbiosis increases the benefits of plant facilitative interactions. Ecography 41: $1-9$.

Montesinos-Navarro A, Verdu M, Querejeta JI, Sortibran L, Valiente-Banuet A. 2016b. Soil fungi promote nitrogen transfer among plants involved in longlasting facilitative interactions. Perspectives in Plant Ecology, Evolution and Systematics 18:45-51.

Montesinos-Navarro A, Verdu M, Querejeta JI, Valiente-Banuet A. 2017. Nurse plants transfer more nitrogen to distantly related species. Ecology 98: 13001310.

Nara K. 2006. Ectomycorrhizal networks and seedling establishment during early primary succession. New Phytologist 169: 169-178.

Nara K, Hogetsu T. 2004. Ectomycorrhizal fungi on established shrubs facilitate subsequent seedling establishment of successional plant species. Ecology 85: 1700-1707.

Newman EI. 1988. Mycorrhizal links between plants: their functioning and ecological significance. Advances in Ecological Research 18:

243-271.

Novotny V, Drozd P, Miller SE, Kulfan M, Janda M, Basset Y, Weiblen GD. 2006. Why are there so many species of herbivorous insects in tropical rainforests? Science 313: 1115-1118.

Onguene N, Kuyper T. 2002. Importance of the ectomycorrhizal network for seedling survival and ectomycorrhiza formation in rain forests of south Cameroon. Mycorrhiza 12: 13-17.

Paine CET, Norden N, Chave J, Forget P-M, Fortunel C, Dexter KG, Baraloto C. 2012. Phylogenetic density dependence and environmental filtering predict seedling mortality in a tropical forest. Ecology Letters 15: 34-41.

Paradis E, Claude J, Strimmer K. 2004. APE: analyses of phylogenetics and evolution in R language. Bioinformatics 20: 289-290.

Parker IM, Gilbert GS. 2004. The evolutionary ecology of novel plant-pathogen interactions. Annual Review of Ecology, Evolution, and Systematics 35: 675-700.

Pausas JG, Verdu M. 2010. The jungle of methods for evaluating phenotypic and phylogenetic structure of communities. BioScience 60: 614-625.

Pickles BJ, Wilhelm R, Asay AK, Hahn AS, Simard SW, Mohn WW. 2017. Transfer of ${ }^{13} \mathrm{C}$ between paired Douglas-fir seedlings reveals plant kinship effects and uptake of exudates by ectomycorrhizas. New Phytologist 214: 400411.

Pozo MJ, Azcon-Aguilar C. 2007. Unraveling mycorrhiza-induced resistance. Current Opinion in Plant Biology 10: 393-398.

Qian H, Jin Y. 2016. An updated megaphylogeny of plants, a tool for generating plant phylogenies and an analysis of phylogenetic community structure. Journal of Plant Ecology 9: 233-239. 
R Core Team. 2015. R: A language and environment for statistical computing, v.3.5.2. Vienna, Austria: Foundation for Statistical Computing.

Reinhart KO, Anacker BL. 2014. More closely related plants have more distinct mycorrhizal communities. Annals of Botany 6: plu051.

Ren L, Lou Y, Zhang N, Zhu X, Hao W, Sun S, Shen Q, Xu G. 2013. Role of arbuscular mycorrhizal network in carbon and phosphorus transfer between plants. Biology and Fertility of Soils 49: 3-11.

Rolhauser AG, Pucheta E. 2017. Directional, stabilizing, and disruptive trait selection as alternative mechanisms for plant community assembly. Ecology 98: 668-677.

Selosse M-A, Richard F, He X, Simard SW. 2006. Mycorrhizal networks: des liaisons dangereuses? Trends in Ecology \& Evolution 21: 621-628.

Song YY, Simard SW, Carroll A, Mohn WW, Zeng RS. 2015. Defoliation of interior Douglas-fir elicits carbon transfer and stress signalling to ponderosa pine neighbors through ectomycorrhizal networks. Scientific Reports 5: 8495.

Stock WD, Verboom GA. 2012. Phylogenetic ecology of foliar N and P concentrations and N: Pratios across mediterranean-type ecosystems. Global Ecology and Biogeography 21: 1147-1156.

Tedersoo L, Jairus T, Horton BM, Abarenkov K, Suvi T, Saar I, Kõljalg U. 2008. Strong host preference of ectomycorrhizal fungi in a Tasmanian wet sclerophyll forest as revealed by DNA barcoding and taxon-specific primers. New Phytologist 180: 479-490.

Teste FP, Simard SW, Durall DM, Guy RD, Jones MD, Schoonmaker AL. 2009. Access to mycorrhizal networks and roots of trees: importance for seedling survival and resource transfer. Ecology 90: 2808-2822.

de Toledo Castanho C, Lortie CJ, Zaitchik B, Prado PI. 2015. A meta-analysis of plant facilitation in coastal dune systems: responses, regions, and research gaps.PeerJ3:e768.

Valiente-Banuet A, Verdu M. 2007. Facilitation can increase the phylogenetic diversity of plant communities. Ecology Letters 10: 1029-1036.

Valiente-Banuet A, Verdu M. 2008. Temporal shifts from facilitation to competition occur between closely related taxa. Journal of Ecology 96: 489-494.

Valiente-Banuet A, Verdu M. 2013. Plant facilitation and phylogenetics. Annual Review of Ecology, Evolution, and Systematics 44: 347-366.

Verd'u M, Gomez-Aparicio L, Valiente-Banuet A. 2012. Phylogenetic relatedness as a tool in restoration ecology: a meta-analysis. Proceedings of the Royal Society of London. Series B, Biological Sciences 279: 1761-1767.

Verd'u M, Jordano P, Valiente-Banuet A. 2010. The phylogenetic structure of plant facilitation networks changes with competition. Journal of Ecology 98: 1454-1461.

Verdu M, Rey PJ, Alcantara JM, Siles G, Valiente-Banuet A. 2009. Phylogenetic signatures of facilitation and competition in successional communities. Journal of Ecology 97: 1171-1180.
Veresoglou SD, Rillig MC. 2014. Do closely related plants host similar arbuscular mycorrhizal fungal communities? A meta-analysis. Plant and Soil 377: 395-406.

Viechtbauer W. 2015. The comprehensive R Archive Network. Package "metafor". [WWW document] URL http://cran. r-project. org/web/packages/metafor/ metafor.pdf [accessed 6 May 2019].

Watt AS. 1947. Pattern and process in the plant community. Journal of Ecology 35: $1-22$.

Webb CO, Gilbert GS, Donoghue MJ. 2006. Phylodiversity-dependent seedling mortality, size structure, and disease in a Bornean rain forest. Ecology 87: S123S131.

Yang X, Chi X, Ji C, Liu H, Ma W, Mohhammat A, Shi Z, Wang X, Yu S, Yue $\mathrm{M}$ et al. 2015. Variations of leaf N, P concentrations in shrubland biomes across northern China: phylogeny, climate and soil. Biogeosciences Discussions 12: $18973-18998$.

Zanne AE, Tank DC, Cornwell WK, Eastman JM, Smith SA, FitzJohn RG, McGlinn DJ, O’Meara BC, Moles AT, Reich PB et al. 2014. Three keys to the radiation of angiosperms into freezing environments. Nature 506: 89-92.

\section{Supporting Information}

Additional Supporting Information may be found online in the Supporting Information section at the end of the article.

Fig. S1 Comparison between the distribution of the phylogenetic distances between plant species used in this study and those reported in facilitation literature.

Table S1 Dataset including the pair-wise plant-plant interac- tions used in this study, the references from which they were obtained and other plant-plant interactions compiled from facil- itation literature to contextualise the dataset. 